\title{
Detection of the SHV genotype polymorphism of the extended-spectrum $\beta$-lactamase-producing Gram-negative bacterium
}

\author{
JUNLONG LI $^{1 *}$, XIAOLI JI ${ }^{1 *}$, XIAOHUI DENG ${ }^{2}$, YINGFENG ZHOU ${ }^{3}$, XIAOQING NI ${ }^{1}$ and XIAOKANG LIU ${ }^{1}$ \\ ${ }^{1}$ Department of Pharmacology, West China School of Preclinical and Forensic Medicine, \\ Sichuan University, Chengdu, Sichuan 610041; ${ }^{2}$ College of Basic Medicine, Xinxiang Medical University; \\ ${ }^{3}$ First Affiliated Hospital, Xinxiang Medical University, Xinxiang, Henan 453003, P.R. China
}

Received November 4, 2014; Accepted December 18, 2014

DOI: $10.3892 /$ br.2015.416

\begin{abstract}
The prevalence of extended-spectrum $\beta$-lactamases (ESBLs) is due to the extensive usage of the extended-spectrum cephalosporins and leads to huge financial loss worldwide, whilst presenting a challenge to the clinical treatment. The aim of the present study was to delineate the frequency of ESBL occurrence in Enterobacteriaceae and confirm the SHV genotype. A random collection of 153 Escherichia coli isolates (E. coli) and 70 Klebsiella pneumoniae isolates were tested. The amplification products obtained by polymerase chain reaction were sequenced. Isolates with novel mutations were transformed to $E$. coli $\mathrm{DH} 5 \alpha$. The minimum inhibitory concentration (MIC) was obtained by a microdilution method. The relevance ratio of ESBL was $67.7 \%$ and the proportion of the SHV $\beta$-lactamase gene $\left(b l a_{\mathrm{SHV}}\right)$ was $18.5 \%$. A new genotype of $\beta$-lactamase was demonstrated and submitted to GenBank. A total of 12 mutational sites were found in $28 \mathrm{ESBL}$-producing isolates, including four nonsense mutations. Sensitive-rates of $28 \mathrm{ESBL}$-producing isolates to imipenem were $100 \%$, and resistant-rates to penicillin, amoxicillin and oxacillin were $100 \%$. The MIC of DH5 $\alpha-\mathrm{F} 8$ to penicillin, oxacillin, cefoxitin, cefotaxime, cefepime, cefoperazone/sulbactam, imipenem and netilmicin was 512, 512, 2, $0.03,0.06,4,0.015$ and 32 respectively. In conclusion, ESBL and SHV-28 is the most prevalent bla. Imipenem is the most effective antibiotic to ESBL, and the 4th-generation cephalosporins and $\beta$-lactamase inhibitor compound are also effective. ESBL
\end{abstract}

Correspondence to: Professor Xiaokang Liu, Department of Pharmacology, West China School of Preclinical and Forensic Medicine, Sichuan University, 3-17 Renmin South Road, Chengdu, Sichuan 610041, P.R. China

E-mail: liuxiaokang6@163.com

${ }^{*}$ Contributed equally

Key words: Escherichia coli, Klebsiella pneumoniae, extended-spectrum $\beta$-lactamase, SHV genotype, mutation, transconjugation, antibiotic sensitivity is mediated by plasmids and able to spread among different Enterobacteriaceae. In conclusion, new mutations of the $b l a_{\mathrm{SHV}}$ gene exist from at least 2010.

\section{Introduction}

Extended-spectrum $\beta$-lactamases (ESBLs) are the plasmid-mediated bacterial enzymes that have been found in enteric Gram-negative bacteria and are important pathogens in nosocomial infections (1). The importance of ESBLs in the spread of multiple resistances among Gram-negative bacteria was thoroughly recognized (2). The majority of ESBLs are derived from broad-spectrum $\beta$-lactamases, TEM-1 and SHV-1. CTX-M and OXA-type enzymes are novel families of ESBLs (3). There are nine distinct structural families, TEM, SHV, CTX-M, PER, VEB, GES, TLA, BES and OXA, which are classified based on comparisons of deduced amino acid sequences (4). TEM and SHV enzymes were included in the first ESBL variant and they remain in the most prevalent types of ESBL (5-7). The first SHV type of an ESBL isolate was reported in Germany in 1983 (8). SHV is one of the most common genotypes of ESBL $(9,10)$.

Recently, ESBLs have increased in prevalence due to the use of extended-spectrum cephalosporins (ESC). The resistance to ESC among Enterobacteriaceae has occurred worldwide (11). $\beta$-lactams were the most frequently prescribed antibiotics accounting for $\sim 50 \%$ of global antibiotic administration. Therefore, ESBLs presented a big challenge to treatment (12). ESBL-producing isolates caused outbreaks of infections that lead to serious antibiotic management concerns with significant economic causatives (13). The main predictor of mortality caused by ESBL-producing isolates was initially inadequate antimicrobial treatment (14). Escherichia coli (E. coli) and Klebsiella pneumoniae (K. pneumoniae) were the most popular ESBL-producing isolates (15). In addition, ESBLs have been found in other Enterobacteriaceae and Pseudomonas aeruginosa (3).

The resistance to the 3rd-generation cephalosporins was mainly due to the production of ESBLs enzymes, which are hydrolyzing oxyimino-cephalosporins inhibited by clavulanic acid (16). ESBL enzymes, such as SHV, TEM and CTX-M, were 
all mediated by the specific plasmids. Conjugation experiments and plasmid identification were conducted to examine the transferability of resistance to ESCs (11). Traditionally, the scope of the problem caused by ESBLs was assessed by considering the microbiological width (the type of microorganisms involved), antibiotic depth (the number of antibiotics hydrolyzed by ESBL) and geographical breadth (the global burden of resistance). Otherwise, the clinical impact of antibiotic resistance, such as the effect on mortality, was extremely difficult to define (1).

To delineate the trends of ESBL-producing isolates, the frequency of SHV in Chengdu (China) were detected by assessing the clinical isolates obtained from hospitals in Chengdu between 2010 and 2011. Confirmation testing of ESBLs-producing isolates was performed by the double-disk synergy test. The minimum inhibitory concentration (MIC) of the ESBLs-producing isolates to antibiotics was obtained by a microdilution method. Polymerase chain reaction (PCR) and DNA sequencing measurement were also performed to determine the genotypes and mutations of the SHV $\beta$-lactamase gene $\left(b l a_{\mathrm{SHV}}\right)$. Additionally, we hypothesized that the ESBL gene may be transferred among Enterobacteriaceae.

\section{Materials and methods}

Bacterial isolates. A total of 223 isolates, including E. coli $(\mathrm{n}=153)$ and $K$. pneumoniae $(\mathrm{n}=70)$, were collected from hospitals in Chengdu between 2010 and 2011 (all the patients provided written consent). The ethics committee of Sichuan University approved the study. E. coli ATCC25922, K. pneumoniae ATCC700603 and a SHV E. coli isolate were used as quality control strains.

Screening and confirmation for ESBL. The ESBL phenotype screening test was performed according to the disk diffusion method recommended by the National Committee for Clinical Laboratory Standards (NCCLS) on Mueller-Hinton agar (Sigma-Aldrich, St. Louis, MO, USA) using commercial antibiotic disks. The antibiotics used were ceftazidime, aztreonam, cefotaxime and ceftriaxone (all $30 \mu \mathrm{g} / \mathrm{disk}$; all Binhe Microorganism Reagent Co., Ltd., Hangzhou, Zhejiang, China). The size of the inhibition holes was measured after the Mueller-Hinton plates (Aoboxing Bio-Tech Co., Ltd., Beijing, China) were incubated at $35^{\circ} \mathrm{C}$ for $16 \mathrm{~h}$. When the size of the inhibition hole was $22 \mathrm{~mm}$ for ceftazidime, $\leq 27 \mathrm{~mm}$ for aztreonam, $\leq 27 \mathrm{~mm}$ for cefotaxime or $\leq 25 \mathrm{~mm}$ for ceftriaxone, the occurrance of any of these circumstances showed the possibility of ESBLs. The double-disk synergy testing method was performed to confirm ESBL. The antibiotics that were used for confirmation testing in ESBLs were cefotaxime (30 $\mu \mathrm{g} / \mathrm{disk})$, cefotaxime-clavulanate (30 $\mu \mathrm{g} / \mathrm{disk})$ (Binhe), ceftazidime (30/10 $\mu \mathrm{g} /$ disk) and ceftazidime-clavulanate $(30 / 10 \mu \mathrm{g} / \mathrm{disk})$ (Binhe Microorganism Reagent Co., Ltd.). When the difference of diameter in any pair of disks was $\geq 5 \mathrm{~mm}$, the isolate was confirmed to produce ESBLs.

Extracting the plasmid DNA and amplification of ESBL genes. The plasmid DNA of ESBL-producing isolates was extracted with the Plasmid Mini Kit I (Omega Bio-Tek, Norcross, GA, USA) and stored at $-20^{\circ} \mathrm{C}$. The presence of $\beta$-lactamase genes was investigated by PCR (Bio-Rad, Hercules, CA, USA). The primers aiming to amplify the SHV genes were designed by the Primer Premier 5.0 (PREMIER Biosoft, Palo Alto CA, USA) according to the original SHV gene sequence from GenBank; accession no. AF124984. The primers were as follows: $b l a_{\mathrm{SHV} 1}$, 5'CTTATTTGTCGCTTC TTT3'; and bla $a_{\mathrm{SHV} 2}$ 5'TTATGGCGTTACCTTTG3'. In the $25 \mu \mathrm{l}$ PCR system was $1 \mu \mathrm{l}(10 \mathrm{pmol} / \mu \mathrm{l})$ primer $b l a_{\mathrm{SHV} 1}, 1 \mu \mathrm{l}(10 \mathrm{pmol} / \mu \mathrm{l})$ primer $b l a_{\mathrm{SHV} 2}, 1 \mu \mathrm{l}(10 \mathrm{ng} / \mu \mathrm{l})$ plasmid, $12.5 \mu \mathrm{l} 2 \mathrm{X}$ Taq Master mix (Tiangen Biotech, Co., Ltd., Beijing, China) and $9.5 \mu \mathrm{l}$ sterile water. PCR amplification was performed under the following conditions: Initial denaturation at $94^{\circ} \mathrm{C}$ for $3 \mathrm{~min}$, denaturation at $94^{\circ} \mathrm{C}$ for $45 \mathrm{sec}$, annealed at $58^{\circ} \mathrm{C}$ for $45 \mathrm{sec}$ and extended at $72^{\circ} \mathrm{C}$ for $1 \mathrm{~min}$. After 35 cycles, the amplification was finally extended at $72^{\circ} \mathrm{C}$ for $7 \mathrm{~min}$. The PCR products were detected by $2 \%$ agarose gel electrophoresis (Sigma-Aldrich).

Transconjugation experiments. The amplification products of $K$. pneumoniae F8, including novel mutations, and $K$. pneumoniae $\mathrm{F} 25$, including the $b l a_{\mathrm{SHV}-18}$ gene to the carrier $\mathrm{pUCm}-\mathrm{T}$ (Sangon Biotech, Shanghai, China) with ampicillin-resistance, were conjugated. The carrier, including the target segments, were transformed to competent cell: E. coli DH5 $\alpha$ (Sangon Biotech). The bacterial strains transformed were selected on Mueller-Hinton agar with ampicillin-resistance with a concentration of $50 \mu \mathrm{g} / \mathrm{ml}$ ampicillin (Binhe Microorganism Reagent Co., Ltd.). The combinations are known as DH5 $\alpha-\mathrm{F} 8$ and DH5 $\alpha-\mathrm{F} 25$ in the following section. The plasmid DNA of DH5 $\alpha-\mathrm{F} 8$ and DH5 $\alpha-\mathrm{F} 25$ were extracted after incubation at $35^{\circ} \mathrm{C}$ for $16-18 \mathrm{~h}$. The plasmids were amplified and primer $b l a_{\mathrm{SHV} 1}$ and $b l a_{\mathrm{SHV} 2}$ were analyzed with $2 \%$ agarose gel electrophoresis (Sigma-Aldrich).

Drug-sensitivity testing of ESBL-producing isolates, $D H 5 \alpha-F 8$ and $D H 5 \alpha-F 25$. The MIC was obtained by the microdilution method according to the standards for antimicrobial susceptibility testing (NCCLS document M100-S9, 2008). The antibiotic concentrations were 512, 256, 128, 64, 32, 16, 8, 4, $2,1,0.5,0.25,0.125,0.06,0.03$ and $0.015 \mathrm{mg} / 1$, respectively. The antibiotics used were penicillin, amoxicillin, oxacillin, cefoxitin, ceftazidime, ceftriaxone, cefotaxime, cefoperazone, cefepime, ampicillin-sulbactam, piperacillin-tazobactam, cefoperazone-sulbactam, imipenem, aztreonam, netilmicin, levofloxacin and gentamicin (all Binhe Microorganism Reagent Co., Ltd.).

\section{Results}

Detection of ESBLs. A total of 151 ESBLs were detected in $112(74.67 \%)$ E. coli and $39(55.7 \%)$ of $K$. pneumoniae isolates. The total relevance ratio was $67.7 \%$. PCR products were $\sim 1,021$ base pairs and there were 28 isolates, including $b l a_{\mathrm{SHV}}$ gene in $16 \mathrm{E}$. coli and $12 \mathrm{~K}$. pneumoniae isolates. The proportion of gene $b l a_{\mathrm{SHV}}$ was $18.5 \%$.

Blast search of $S H V$. The results of Blast searches revealed that there were nine isolates of SHV-28, seven of SHV-11, four of SHV-1, three of SHV-12, one of SHV-31 and one of SHV-18. There were another three isolates that included novel mutations and their nucleotide sequence had no difference between each other and cannot be found in the NCBI reference genome. The 
Table I. Mutations of the known SHV genotypes of bla.

\begin{tabular}{lccccr}
\hline Mutation sites & $\begin{array}{c}\text { Quondam } \\
\text { bases }\end{array}$ & $\begin{array}{c}\text { Bases after } \\
\text { mutation }\end{array}$ & $\begin{array}{c}\text { Quondam } \\
\text { amino acid }\end{array}$ & $\begin{array}{c}\text { Amino acid } \\
\text { after mutation }\end{array}$ & $\begin{array}{c}\text { Mutational } \\
\text { rates (\%) }\end{array}$ \\
\hline $7($ Y7F) & A & T & Tyr & Phe & 32.1 \\
$8(\mathrm{I} 8 \mathrm{~F})$ & $\mathrm{A}$ & $\mathrm{T}$ & $\mathrm{IIe}$ & Phe & 3.6 \\
$35(\mathrm{~L} 35 \mathrm{Q})$ & $\mathrm{T}$ & $\mathrm{A}$ & Leu & Gln & 39.2 \\
$43(\mathrm{R} 43 \mathrm{~S})$ & $\mathrm{C}$ & $\mathrm{A}$ & Arg & Ser & 3.6 \\
$238(\mathrm{G} 238 \mathrm{~S}$ or G238A) & $\mathrm{G}$ & A or C & Gly & Ser or Ala & $10.7,3.6$ \\
$240(\mathrm{E} 240 \mathrm{~K})$ & $\mathrm{G}$ & $\mathrm{A}$ & Glu & Lys & 17.9 \\
\hline
\end{tabular}

Table II. Results of 28 extended-spectrum $\beta$-lactamases (ESBL)-producing isolates to 17 antibiotics.

\begin{tabular}{lccc}
\hline & \multicolumn{3}{c}{ 28 ESBL-producing strains } \\
\cline { 2 - 4 } & $\begin{array}{c}\text { Resistance } \\
(\%)\end{array}$ & $\begin{array}{c}\text { Intermediary } \\
\text { Antibiotics }\end{array}$ & $\begin{array}{c}\text { Sensitive } \\
(\%)\end{array}$ \\
\hline Oxacillin & 100.0 & 0.0 & 0.0 \\
Penicillin & 100.0 & 0.0 & 0.0 \\
Amoxicillin & 100.0 & 0.0 & 0.0 \\
Cefoxitin & 95.9 & 4.1 & 0.0 \\
Cefotaxime & 92.7 & 0.0 & 8.3 \\
Ceftazidime & 45.8 & 16.7 & 37.5 \\
Cefoperazone & 87.5 & 4.1 & 8.3 \\
Ceftriaxone & 92.7 & 0.0 & 8.3 \\
Cefepime & 11.0 & 12.5 & 76.5 \\
Amoxicillin/clavulanate & 83.4 & 8.3 & 8.3 \\
Piperacillin/tazobactam & 8.3 & 16.6 & 75.0 \\
Cefoperazone/sulbactam & 4.2 & 12.5 & 83.3 \\
Aztreonam & 59.9 & 0.0 & 40.1 \\
Imipenem & 0.0 & 0.0 & 100.0 \\
Levofloxacin & 66.7 & 8.3 & 33.0 \\
Netilmicin & 37.5 & 20.8 & 41.7 \\
Gentamicin & 40.3 & 8.3 & 33.0 \\
\hline & & &
\end{tabular}

novel sequence was submitted to GenBank with an accession no. JX192924. The gene sequence used, was: 1 ctttgggctc ctcatgatgt attgtg gtta tgcgtcatat tcgectgtgt attatctcce 61 tgttagccac cetgccgetg gcggtacaag ccagcccgca gecgettgag caaattaaac 121 aaagcgaaag ccagctgtcg ggccgcgtag gcatgataga aatggatctg gccagcggcc 181 gcacgctgac cgcetggcgc gccgatgaac gcttcccat gatgagcacc tttaaagtag 241 tgctctgcgg cgcagtgctg gcgcgggtgg atgccggtga cgaacagctg gagcgaaaga 301 tccactatcg ccagcaggat ctggtggact actcgccggt cagcgaaaaa caccttgccg 361 acggcatgac ggtcggcgaa ctctgcgecg cegccattac catgagcgat aacagcgecg 421 ccaatctget getggecacc gtcggcggec ccgcaggatt gactgecttt ttgcgccaga 481 tcggcgacaa cgttacccge cttgaccget gggaaacgga actgaatgag gegcttcceg 541 gegacgcccg cgacaccact accceggcca gcatggcegc gaccetgcgc aagctgctga 601 ccagccagcg tctgagcgec cgttcgcaac ggcagctgct gcagtggatg gtggacgatc 661 gggtcgccgg accgttgatc cgctcegtgc tgceggeggg ctggtttatc gecgataaga 721 ccggagctgg cgaacggggt gegcgeggga ttgtcgecet gettggeccg

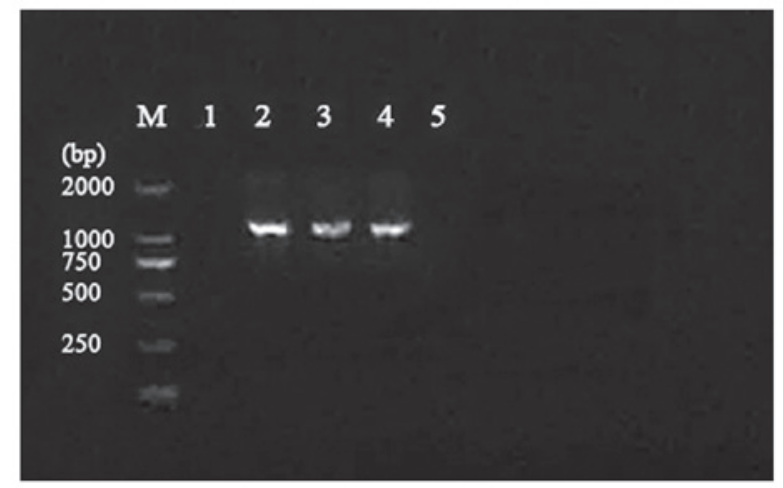

Figure 1. Plasmid DNA of DH5 $\alpha-\mathrm{F} 8$ and DH5 $\alpha-\mathrm{F} 28$ was observed by agarose gel electrophoresis. The plasmid was successully transformed. Lane M, marker; 1, positive control; 2, SHV positive strains; 3 , DH5 $\alpha$-F8; 4 , DH $5 \alpha-F 25 ; 5$, SHV negative strains.

aataacaaag 781 cagagcgcat tgtggtgatt tatctgcggg atacgccggc gagcatggec gagcgaaatc 841 agcaaatcge cgggatcgge gcggcgetga tcgagcactg gcaacgctaa cccggcggtg 901 gcgcgcgcgt tatccggctc gtagcactcg cagctgccgg gcgatatgac tggcggcggc 961 atcggagaga tgccgtcggt aatgatggtg gtgaaccggg tcaaagtatc accccataa $1021 \mathrm{c}$; the coding region was from 33 to 893 .

These five known genotypes of $b l a_{\mathrm{SHV}}$ included 10 mutational sites. However, these changes do not lead to amino acid changes at position of 112, 260, 268 and 274, which were so-called nonsense mutations. Other mutations are represented in Table I. Three isolates, including novel mutations, contain five mutational sites: Base 121 at amino acid position 35 changes from ' $\mathrm{T}$ ' to ' $\mathrm{A}$ ' and the amino acid Leu is substituted by Gln; recorded as L35Q in GenBank. Base 36 in the coding region changes from ' $\mathrm{T}$ ' to ' $\mathrm{C}$ ', with a Tyr-to-His substitution; base 89 from ' $\mathrm{C}$ ' to 'A', with a His-to-Gln substitution; base 353 from ' $\mathrm{T}$ ' to ' $\mathrm{C}$ ' and base 494 from ' $\mathrm{C}$ ' to ' $\mathrm{T}$ ', but the amino acid of these two positions does not change.

Extraction of DH5 $\alpha$ plasmids. The plasmid DNA of DH5 $\alpha-\mathrm{F} 8$ and DH5 $\alpha-$ F2 8 were extracted, as shown in Fig. 1. The results showed that the gene sequence was identical to that of JX192924. This accounted for the gene segments with novel mutations successfully transconjugating to E. coli DH5 $\alpha$. There are three mutational amino acids that differ from the original (accession no. EU441172); H3F, Q20H and Q31L. Additionally, this mutation has not emerged since the first SHV-28 strain was detected. 
Table III. Minimum inhibitory concentration (MIC) of the transconjugation isolates, $\mathrm{DH} 5 \alpha-\mathrm{F} 8$ and $\mathrm{DH} 5 \alpha-\mathrm{F} 25$, to 17 antibiotics.

\begin{tabular}{lcc}
\hline & \multicolumn{2}{c}{ MIC, mg/l } \\
\cline { 2 - 3 } & DH5 $\alpha$-F8 & \\
& L35Q & DH5 $\alpha$-F25 \\
Antibiotics & 89th base & G238A \\
\hline Oxacillin & 512 & 512 \\
Penicillin & 512 & 512 \\
Amoxicillin & 256 & 256 \\
Cefoxitin & 2 & 4 \\
Cefotaxime & 0.03 & 4 \\
Ceftazidime & 0.25 & 8 \\
Cefoperazone & 4 & 32 \\
Ceftriaxone & 0.125 & 0.25 \\
Cefepime & 0.06 & 0.06 \\
Amoxicillin/clavulanate & 32 & 16 \\
Piperacillin/tazobactam & 64 & 64 \\
Cefoperazone/sulbactam & 4 & 4 \\
Aztreonam & 16 & 8 \\
Imipenem & 0.015 & 0.015 \\
Levofloxacin & 64 & 0.03 \\
Netilmicin & 32 & 0.5 \\
Gentamicin & 128 & 0.06 \\
\hline
\end{tabular}

Antimicrobial susceptibility testing. The results of the antimicrobial susceptibility testing are shown in Table II. The MIC of DH5 $\alpha-\mathrm{F} 8$ and DH5 $\alpha-\mathrm{F} 25$ to 17 antibiotics are shown in Table III. A total of 28 ESBL-producing isolates were all sensitive to imipenem, and all resistant to penicillin, amoxicillin and oxacillin. The sensitive-rates to cefoperazone/sulbactam, piperacillin/tazobactam and cefepime were all $>75 \%$. The resistant-rates to the 2 nd and 3rd-generation cephalosporins and levofloxacin were high. DH5 $\alpha-\mathrm{F} 8$ and DH5 $\alpha-F 25$ were resistant to penicillin, amoxicillin and oxacillin, and sensitive to imipenem, the 3rd and 4th-generation cephalosporins and the $\beta$-lactamase inhibitor compound, except for piperacillin/tazobactam. Compared to DH5 $\alpha-\mathrm{F} 25$, DH5 $\alpha-\mathrm{F} 8$ was sensitive to levofloxacin, netilmicin and gentamicin.

\section{Discussion}

Currently, antibiotic resistance is a severe problem. As opposed to methicillin-resistant Staphylococcus aureus and vancomycin-resistant Enterococci, ESBLs-producing strains are another class of primary pathogenic bacteria $(17,18)$. ESBLs mediated by plasmids are common in Enterobacteriaceae, particularly in E. coli and K. pneumoniae, which have been reported to cause serious infections in hospitals, as well as in the community in different parts of the world $(19,20)$. A previous study indicated that the present disk diffusion criteria underestimate the prevalence of ESBL-producing strains (21).
All the phenomena suggest that the trends of ESBLs-producing isolates strains may be underestimated.

SHV-2 is the first reported genotype of bla, which has only one mutation site at codon 238. The change from glycine to serine induces the hydrolysis of the 3rd-generation cephalosporin (15). Subsequent to the identification of SHV-2, numerous types of SHV genotypes have been found successively. One study identified SHV-8 with an Asp-to-Asn substitution at amino acid position 179 in a bacteremia patient that had received multiple courses of antimicrobial agents, including ceftazidime. The collection of blood isolates from the same patient suggested the in vivo evolution of resistance under selective pressure of treatment with cephalosporins (1).

With regards to the high presence ratio in Chengdu of the ESBL SHV genotypes, SHV-1, SHV-11 and SHV-28 were the most popular types. A total of 28 ESBL-producing isolates showed a high resistance-rate to oxacillin, amoxicillin, penicillin, cefoxitin, levofloxacin and gentamicin. Similarly, Jain et al (22) also reported that ESBL-producing organisms were resistant to ampicillin, cotrimoxazole, tetracycline and gentamicin. According to our study, the results indicated that broad-spectrum penicillins, such as amoxicillin and penicillin, were ineffective to the infection of ESBL-producing isolates. In addition, $\beta$-lactamase inhibitor compounds, such as clavulanic acid and sulbactam, are able to enhance the sensitivity of ESBL-producing isolates to $\beta$-lactamase (23). Tazobactam enhanced the antibacterial function of piperacillin as a new type of $\beta$-lactamase inhibitor. In the present study, the drug-sensitivity testing showed that piperacillin/tazobactam was superior to amoxicillin/clavulanate. For the prior use of clavulanate, is is possible that the bacterium was less sensitive compared to tazobactam and sulbactam. The 4th-generation cephalosporin and $\beta$-lactamase inhibitor compound are effective to ESBL, while the carbapenems, such as imipenem, are most available to ESBL. DH5 $\alpha-\mathrm{F} 8$ and DH5 $\alpha-\mathrm{F} 25$ were resistant to penicillin, oxacillin, amoxicillin, amoxicillin/clavulanate, aztreonam and piperacillin/tazobactam, and sensitive to 3rd and 4th-generation cephalosporins and cefoperazone/sulbactam. The MIC of DH5 $\alpha-F 25$ to the 3rd-generation cephalosporin was higher than that of DH5 $\alpha-\mathrm{F} 8$. DH5 $\alpha-\mathrm{F} 25$ includes two mutations at codons 238 and 240. These codons did not mutate in DH5 $\alpha-\mathrm{F} 8$. A previous study reported that mutations at codons 238 and 240 are relevant to the resistance to cefotaxime and ceftazidime (24). The MIC of the original isolates of F8 and F25 showed a high resistant-rate compared to DH5 $\alpha-\mathrm{F} 8$ and DH5 $\alpha-\mathrm{F} 25$, which suggested that the original isolates performed other resistant mechanisms leading to the resistance to the 3rd-generation cephalosporin. Further study is required to confirm their resistant mechanism. Multi-resistance isolates are now common in the clinic, so the choosing of antibiotics should be based on the drug-sensitivity testing. The 3rd-generation cephalosporin should be replaced by cephamycin, a $\beta$-lactamase inhibitor compound and carbapenem.

In conclusion, the present study demonstrated that ESBL was mediated by the plasmids. The ESBL-producing members, such as E. coli and K. pneumoniae, showed a high resistance-rate to numerous types of antibiotics. Novel 
mutations have existed in Chengdu from at least 2010. Further study is required to determine its biochemical character.

\section{References}

1. Rahal JJ: Extended-spectrum beta-lactamases: how big is the problem? Clin Microbiol Infect 6 (Suppl 2): 2-6, 2000.

2. Cohen ML: Epidemiology of drug resistance: implications for a post-antimicrobial era. Science 257: 1050-1055, 1992.

3. Bradford PA: Extended-spectrum beta-lactamases in the 21st century: characterization, epidemiology and detection of this important resistance threat. Clin Microbiol Rev 14: 933-951, 2001.

4. GniadkowskiM:Evolutionandepidemiologyofextended-spectrum beta-lactamases (ESBLs) and ESBL-producing microorganisms. Clin Microbiol Infect 7: 597-608, 2001.

5. Kliebe C, Nies BA, Meyer JF, Tolxdorff-Neutzling RM and Wiedemann B: Evolution of plasmid-coded resistance to broad-spectrum cephalosporins. Antimicrob Agents Chemother 28: 302-307, 1985.

6. Knothe H, Shah P, Krcmery V, Antal M and Mitsuhashi S: Transferable resistance to cefotaxime, cefoxitin, cefamandole and cefuroxime in clinical isolates of Klebsiella pneumoniae and Serratia marcescens. Infection 11: 315-317, 1983.

7. Sirot D, Sirot J, Labia R, et al: Transferable resistance to third-generation cephalosporins in clinical isolates of Klebsiella pneumoniae: identification of CTX-1, a novel beta-lactamase. J Antimicrob Chemother 20: 323-334, 1987.

8. Coudron PE, Moland ES and Sanders CC: Occurrence and detection of extended-spectrum beta-lactamases in members of the family Enterobacteriaceae at a veterans medical center: seek and you may find. J Clin Microbiol 35: 2593-2597, 1997.

9. Tzouvelekis LS and Bonomo RA: SHV-type beta-lactamases. Curr Pharm Des 5: 847-864, 1999.

10. Paterson DL, Hujer KM, Hujer AM, et al: Extended-spectrum beta-lactamases in Klebsiella pneumoniae bloodstream isolates from seven countries: dominance and widespread prevalence of SHV- and CTX-M-type beta-lactamases. Antimicrob Agents Chemother 47: 3554-3560, 2003.

11. Shaheen BW, Nayak R, Foley SL, et al: Molecular characterization of resistance to extended-spectrum cephalosporins in clinical Escherichia coli isolates from companion animals in the United States. Antimicrob Agents Chemother 55: 5666-5675, 2011.

12. Livermore DM: Beta-lactamase-mediated resistance and opportunities for its control. J Antimicrob Chemother 41 (Suppl D) 25-41, 1998.
13. Jonathan N: Screening for extended-spectrum beta-lactamase-producing pathogenic enterobacteria in district general hospitals. J Clin Microbiol 43: 1488-1490, 2005.

14. Oteo J, Perez-Vazquez M and Campos J: Extended-spectrum [beta]-lactamase producing Escherichia coli: changing epidemiology and clinical impact. Curr Opin Infect Dis 23: 320-326, 2010.

15. Du Bois SK, Marriott MS and Amyes SG: TEM- and SHV-derived extended-spectrum beta-lactamases: relationship between selection, structure and function. J Antimicrob Chemother 35: 7-22, 1995

16. Canton R and Coque TM: The CTX-M beta-lactamase pandemic Curr Opin Microbiol 9: 466-475, 2006.

17. Jacoby GA: Epidemiology of extended-spectrum beta-lactamases. Clin Infect Dis 27: 81-83, 1998.

18. Dashti AA, Jadaon MM, Gomaa HH, Noronha B and Udo EE: Transmission of a Klebsiella pneumoniae clone harbouring genes for CTX-M-15-like and SHV-112 enzymes in a neonatal intensive care unit of a Kuwaiti hospital. J Med Microbiol 59: 687-692, 2010

19. Pitout JD, Nordmann P, Laupland KB and Poirel L: Emergence of Enterobacteriaceae producing extended-spectrum beta-lactamases (ESBLs) in the community. J Antimicrob Chemother 56: 52-59, 2005.

20. Huang SS, Lee MH and Leu HS: Bacteremia due to extended-spectrum beta-lactamase-producing Enterobacteriaceae other than Escherichia coli and Klebsiella. J Microbiol Immunol Infect 39: 496-502, 2006.

21. Huijbers PM, Graat EA, Haenen AP, et al: Extended-spectrum and AmpC $\beta$-lactamase-producing Escherichia coli in broilers and people living and/or working on broiler farms: prevalence, risk factors and molecular characteristics. J Antimicrob Chemother 69: 2669-2675, 2014.

22. Jain A, Roy I, Gupta MK, Kumar M and Agarwal SK: Prevalence of extended-spectrum beta-lactamase-producing Gram-negative bacteria in septicaemic neonates in a tertiary care hospital. J Med Microbiol 52: 421-425, 2003.

23. Jacoby GA and Carreras I: Activities of beta-lactam antibiotics against Escherichia coli strains producing extended-spectrum beta-lactamases. Antimicrob Agents Chemother 34: 858-862, 1990.

24. Harada S, Ishii Y and Yamaguchi K.: Extended-spectrum beta-lactamases: implications for the clinical laboratory and therapy. Korean J Lab Med 28: 401-412 2008. 